\title{
DISCONTINUOUS SEMILINEAR DIFFERENTIAL EQUATIONS AND MULTIPLE VALUED MAPS
}

\author{
JEFFREY RAUCH ${ }^{1}$
}

\begin{abstract}
To treat boundary value problems with nonlinearity of the form $\boldsymbol{g}(u)$ where $g \in L_{\infty}^{\operatorname{loc}}(R)$ one must extend $g$ to be multiple valued. Once this is done weak solutions are obtained.
\end{abstract}

1. Introduction. This paper discusses semilinear differential equation with nonlinear term, $g(u)$, involving a discontinuous function $g$. For definiteness we will consider the example

$$
\begin{aligned}
-\Delta u+g(u)=f & & \text { in } \Omega, \\
u=0 & & \text { in } \partial \Omega,
\end{aligned}
$$

where $\Omega$ is a bounded open set in $\mathbf{R}^{n}$, and $\Delta=\partial^{2} / \partial x_{1}^{2}+\cdots+\partial^{2} / \partial x_{n}^{2}$ is the Laplace operator. When $\boldsymbol{g}$ is monotonically increasing this problem is well understood. One introduces the multiple valued function $\hat{\boldsymbol{g}}(s)$ defined by

$$
\hat{g}(s)=[g(s-), g(s+)] \text {. }
$$

At points of continuity $\hat{\boldsymbol{g}}(s)$ is one number while at points of discontinuity $\hat{\boldsymbol{g}}(s)$ is an entire interval. The graph of $\hat{\boldsymbol{g}}$ is the graph of $g$ with the jumps filled in. It is known that for any $f \in \dot{H}_{1}(\Omega)^{\prime} \equiv H_{-1}(\Omega)$ there is a $u \in \dot{H}_{1}(\Omega)$ and a $\gamma \in H_{-1}(\Omega) \cap L_{1}(\Omega)$ such that

$$
\begin{array}{cl}
-\Delta u+\gamma=f & \text { in } \Omega, \\
\gamma(x) \in \hat{g}(u(x)) & \text { a.e. in } \Omega .
\end{array}
$$

It is in the sense of (3), (4) that one has $-\Delta u+g(u)=f$. One might guess that since $g$ is continuous except at a countable set that the multiple valued property of $g$ is inessential and actually $\gamma=g(u)$ a.e. regardless what values $g$ is assigned at the jump. This hope evaporates with the following

EXAMPle. Let $f=0$ and $g(0+)>0>g(0-)$. The solution of (4), (5) is $u \equiv 0$ and it is clearly very important that $g(0) \ni 0$.

In the monotone theory, the necessity of filling in the jumps of $g$ is dictated by general results which emphasize the importance of maximal monotonicity in proving that maps are surjective (see [1, §II.5]). It is the goal of this paper to show that entirely analogous results are true when $g$ is not monotone. One only needs that "ultimately" $g$ has increased, that is $\varlimsup_{s \rightarrow-\infty} g(s)<\underline{\lim }_{s \rightarrow+\infty}$

Received by the editors August 2, 1976.

AMS (MOS) subject classifications (1970). Primary 35J66, 35K55, 35L60.

'Research partially supported by the National Science Foundation under grant NSF GP 34260.

- American Mathematical Society 1977 
$g(s)$. It is also interesting that for $g$ which are not monotone the discontinuities may be more numerous and more varied and the process of "filling in the jumps" is correspondingly more delicate.

Though we only present the details for the elliptic problem (3), (4), analogous results are true for hyperbolic and parabolic equations. We mention some examples at the end of the paper.

2. Filling in the jumps. Suppose $g: \mathbf{R} \rightarrow \mathbf{R}$ is in $L_{\infty}^{\mathrm{loc}}(\mathbf{R})$, that is, the restriction of $g$ to any bounded interval $I$ is in $L_{\infty}(I)$. For any $\varepsilon>0$ and $s \in \mathbf{R}$ let

$$
\bar{g}_{\varepsilon}(s)=\underset{|t-s|<\varepsilon}{\operatorname{ess} \sup } g(s), \quad \underline{g}_{\varepsilon}(s)=\underset{|t-s|<\varepsilon}{\operatorname{essinf}} g(s) .
$$

For $s$ fixed $\bar{g}(s)$ is a decreasing function of $\varepsilon$ and $\underline{g}$ is increasing in $\varepsilon$. Let

$$
\bar{g}(s)=\lim _{\varepsilon \rightarrow 0} \bar{g}_{\varepsilon}(s), \quad \underline{g}(s)=\lim _{\varepsilon \rightarrow 0} \underline{g}_{\varepsilon}(s) .
$$

The function $\bar{g}$ is upper semicontinuous and $\underline{g}$ is lower semicontinuous.

EXAMPLE. If $g$ has a jump discontinuity at $s$ then

$$
\bar{g}(s)=\max (g(s+), g(s-)), \quad \underline{g}(s)=\min (g(s+), g(s-)) .
$$

We define a multiple valued function by

$$
\hat{\mathbf{g}}(s)=[\underline{g}(s), \bar{g}(s)] .
$$

TheOREM. Suppose $g: \mathbf{R} \rightarrow \mathbf{R}$ is in $L_{\infty}^{\mathrm{loc}}$ and for some $s$,

$$
\underset{(-\infty,-s)}{\text { ess sup }} g \leqslant \underset{(s, \infty)}{\operatorname{essinf}} g \text {. }
$$

For any $f \in \stackrel{\circ}{H}_{1}(\Omega)^{\prime}$ there is a $u \in \stackrel{\circ}{1}_{1}(\Omega)$ and $a \gamma \in \stackrel{\circ}{H}_{1}(\Omega)^{\prime} \cap L_{1}(\Omega)$ such that

$$
-\Delta u+\gamma=f \text { in } \Omega \text {, }
$$

and

$$
\gamma(x) \in \hat{g}(u(x)) \text { a.e. in } \Omega \text {. }
$$

Proof. Choose a number $\alpha$ such that $g(-t)<\alpha<g(t)$ for almost all $t$ with $|t|>s$. Replacing $g$ by $g-\alpha$ and $f$ by $f-\alpha$ we may assume without loss of generality that ess $\sup _{(-\infty, s)} g<0<\operatorname{essinf}_{(s, \infty)} g$.

Choose $j \in C_{0}^{\infty}(-1,1)$ such that $j>0$ and $\int_{-\infty}^{\infty} j(s) d s=1$. Let $j_{n}(s)=$ $n j(n x)$ and $g_{n}=j_{n}^{*} g$. A natural way to attack the problem (6), (7) is to find exact solutions of the perturbed problems $-\Delta u+g_{n}(u)=f$ and consider the limit $n \rightarrow \infty$. To avoid assuming familiarity with the perturbed problem we will use a Galerkin procedure which at the $n$th stage gives an approximate solution to $-\Delta u+g_{n}(u)=f$.

Choose finite dimensional subspaces $V_{n} \subset \stackrel{\circ}{H}_{1}(\Omega) \cap L_{\infty}(\Omega)$ so that $V_{n+1} \supset$ $V_{n}$ and $\cup_{n} V_{n}$ is dense in $\stackrel{\circ}{1}_{1}(\Omega)$. For any $n>0$ define $T_{n}: V_{n} \rightarrow V_{n}^{\prime}$ by

$$
\left\langle T_{n}(u), v\right\rangle=\int_{\Omega} \nabla u \cdot \nabla v+g_{n}(u) v-f v d x
$$

for any $u, v \in V_{n}$. We choose $u_{n} \in V_{n}$ such that $T_{n}\left(u_{n}\right)=0$. To see that this is possible observe that for $u \in V_{n}$. 


$$
\left\langle T_{n}(u), u\right\rangle=\int_{\Omega}|\nabla u|^{2}+u g_{n}(u)-f u d x .
$$

Choose positive numbers $\rho, \mu$ such that for all $n$,

$$
\begin{aligned}
g_{n}(t)>0 & \text { if } t>\rho, \\
g_{n}(t)<0 & \text { if } t<-\rho, \text { and } \\
\left|g_{n}(t)\right|<\mu & \text { if }|t|<\rho .
\end{aligned}
$$

Then,

Thus

$$
\begin{aligned}
\int_{\Omega} u g_{n}(u) & =\int_{|u|>\rho} u g_{n}(u)+\int_{|u|<\rho} u g_{n}(u) \\
& >0-\rho \mu|\Omega|>-\rho \mu|\Omega| .
\end{aligned}
$$

$$
\begin{aligned}
\left\langle T_{n}(u), u\right\rangle & >\|\Delta u\|_{0}^{2}-\rho \mu|\Omega|-\|f\|_{-1}\|u\|_{1} \\
& >c_{1}\|u\|_{1}^{2}-c_{2}\|u\|_{1}-c_{3} .
\end{aligned}
$$

In particular, there is an $r>0$ so that if $\|u\|_{1}>r$ then $\left\langle T_{n}(u), u\right\rangle>0$. It follows from Brouwer's fixed point theorem that for each $n$ there is a $u_{n} \in V_{n}$ with $\left\|u_{n}\right\|_{1}<r$ and $T_{n}\left(u_{n}\right)=0$; see [3, Chapter 1, Lemma 4.3]. By Rellich's compactness criterion we may pass to a subsequence, which we still denote as $u_{n}$, so that

$$
\begin{array}{ll}
u_{n} \rightarrow u & \text { weakly in } \dot{H}_{1}(\Omega), \\
u_{n} \rightarrow u & \text { in } L_{2}(\Omega), \\
u_{n} \rightarrow u & \text { a.e. in } \Omega .
\end{array}
$$

We next investigate the convergence of $g_{n}\left(u_{n}\right)$. Following the idea of Strauss [4], the function $g_{n}\left(u_{n}\right)$ is controlled by estimating $\int_{\Omega}\left|u_{n} g_{n}\left(u_{n}\right)\right|$ :

$$
\begin{aligned}
\int_{\Omega}\left|u_{n} g_{n}\left(u_{n}\right)\right| & =\int_{|u|>\rho} u_{n} g_{n}\left(u_{n}\right)+\int_{\left|u_{n}\right|<\rho}\left|u_{n} g_{n}\left(u_{n}\right)\right| \\
& <\int_{\Omega} u_{n} g_{n}\left(u_{n}\right)+2 \int_{\left|u_{n}\right|<\rho}\left|u_{n} g_{n}\left(u_{n}\right)\right| .
\end{aligned}
$$

Since $T_{n}\left(u_{n}\right)=0$ and $\left\|u_{n}\right\|_{1}<$ const, we have

$$
\int_{\Omega} u_{n} g_{n}\left(u_{n}\right)=\int_{\Omega}-\left|\nabla u_{n}\right|^{2}+f u_{n} d x<\text { const. }
$$

Thus,

$$
\int_{\Omega}\left|u_{n} g_{n}\left(u_{n}\right)\right| \leqslant \text { const }+2 \rho \mu|\Omega| \text {. }
$$

Using this estimate, we show that $\left\{g_{n}\left(u_{n}\right)\right\}$ is weakly precompact in $L_{1}(\Omega)$, that is, for each $\varepsilon>0$ we show that there is a $\delta$ so that if $|\omega|<\delta$ then $\int_{\omega}\left|g_{n}\left(u_{n}\right)\right|<\varepsilon$. We use the simple estimate

$$
\left|g_{n}(s)\right|<k^{-1}\left|s g_{n}(s)\right|+\sup _{|s|<k}\left|g_{n}(s)\right|
$$


Choose $k$ so large that $k^{-1} \int_{\Omega}\left|u_{n} g_{n}\left(u_{n}\right)\right|<\varepsilon / 2$ for all $n$, and then pick $\delta$ so that

$$
\delta \text { ess sup }|g(s)|<\varepsilon / 2
$$

If $|\omega|<\delta$, then

$$
\begin{aligned}
\int_{\omega}\left|g_{n}\left(u_{n}\right)\right| & <\frac{1}{k} \int_{\omega}\left|u_{n} g_{n}\left(u_{n}\right)\right|+\int_{\omega} \sup _{|s|<k}\left|g_{n}(s)\right| \\
& <\frac{1}{k} \int_{\Omega}\left|u_{n} g_{n}\left(u_{n}\right)\right|+\delta \text { ess sup }|g(s)|<\varepsilon .
\end{aligned}
$$

Passing to a subsequence (still denoted $u_{n}$ ) we may assume that $g_{n}\left(u_{n}\right) \rightarrow \gamma$ weakly in $L_{1}(\Omega)$. From the equation $T_{n}\left(u_{n}\right)=0$ we conclude that for all $n>m$ and $v \in V_{m}$ we have

$$
\int_{\Omega} \nabla u_{n} \cdot \nabla v+g_{n}\left(u_{n}\right) v-f v d x=0 .
$$

We may pass to the limit $n \rightarrow \infty$ since $v \in L_{\infty}(\Omega)=L_{1}(\Omega)^{\prime}$ to obtain

$$
\int_{\Omega} \nabla u \cdot \nabla v+\gamma v-f v d x=0 \quad \forall v \in V_{m} .
$$

Since $m$ was arbitrary this holds for all $v \in \cup V_{m}$. In particular,

$$
\left|\int_{\Omega} \gamma v\right|<c\|v\|_{1} \quad \forall v \in \cup V_{m},
$$

so $\gamma \in \dot{H}_{1}(\Omega)^{\prime}$. Therefore, since $\cup V_{m}$ is dense in $\dot{H}_{1}(\Omega)$, the identity (8) extends by continuity to all $v \in \dot{H}_{1}(\Omega)$. Thus $-\Delta u+\gamma=f$.

To finish the proof we must show that $\gamma \in \hat{g}(u)$ a.e. or, equivalently,

$$
\underline{g}(u(x))<\gamma<\bar{g}(u(x)) \quad \text { a.e. in } \Omega \text {. }
$$

Let $\eta>0$ be a positive number. Since $u_{n}$ converges a.e. to $u$ we may choose a set $\omega$ so that $|\omega|<\eta$, and

$$
u_{n} \rightarrow u \text { uniformly on } \Omega \backslash \omega, \quad u \in L_{\infty}(\Omega \backslash \omega) \text {. }
$$

Thus for any $\varepsilon>0$ there is an $n_{0}>2 / \varepsilon$ such that for $n>n_{0},\left|u_{n}(x)-u(x)\right|$ $<\varepsilon / 2$ for all $x \in \Omega \backslash \omega$. If $n>n_{0}$ and $x \in \Omega \backslash \omega$, then $g_{n}\left(u_{n}(x)\right)$ is an average of $g$ over the interval $\left|s-u_{n}(x)\right|<\varepsilon / 2$ which is a subset of the interval $(u(x)-\varepsilon, u(x)+\varepsilon)$. Therefore

$$
\underline{g}(u(x)) \leqslant g_{n}\left(u_{n}(x)\right) \leqslant \bar{g}(u(x)) .
$$

Equivalently, if $h \in L_{\infty}(\Omega \backslash \omega), h \geqslant 0$ a.e. then

$$
\int_{\Omega \backslash \omega} \underline{g}_{\varepsilon}(u(x)) h \leqslant \int_{\Omega \backslash \omega} g_{n}\left(u_{n}(x)\right) h \leqslant \int_{\Omega \backslash \omega} \bar{g}_{\varepsilon}(u(x)) h .
$$

Let $n \rightarrow \infty$ using the weak convergence of $g_{n}\left(u_{n}\right)$ to obtain

$$
\int_{\Omega \backslash \omega} \underline{g}_{\varepsilon}(u(x)) h \leqslant \int_{\Omega \backslash \omega} \gamma h \leqslant \int_{\Omega \backslash \omega} \bar{g}_{\varepsilon}(u(x)) h .
$$


Since $u$ is bounded on $\Omega \backslash \omega$, Lebesgue's theorem allows us to take the limit $\varepsilon \rightarrow 0$ to obtain

$$
\int_{\Omega \backslash \omega} \underline{g}(u(x)) h<\int_{\Omega \backslash \omega} \gamma h<\int_{\Omega \backslash \omega} \bar{g}(u(x)) h .
$$

Since $h>0$ was arbitrary it follows that

$$
\underline{g}(u(x))<\gamma<\bar{g}(u(x)) \text { a.e. } x \in \Omega \backslash \omega .
$$

Since we may choose $\eta$ as small as we like, it follows that

$$
\underline{g}(u)<\gamma<\bar{g}(u) \text { a.e. in } \Omega
$$

and the proof is complete.

3. Some other problems. It is clear that other elliptic operators and different boundary conditions can be treated without essential changes. In addition, examples where the linear elliptic operator has a kernel are mentioned in [2].

It is also true that semilinear hyperbolic and parabolic equations can be handled. For example, we have the following result for a nonlinear wave equation.

THEOREM. If $g \in L_{\infty}^{\text {loc }}(\mathbf{R})$ satisifes (5), then for any $\phi \in \dot{H}_{1}(\Omega), \psi \in L_{2}(\Omega)$ there is a $u \in L_{2}\left([0, T]: \dot{H}_{1}(\Omega)\right) \cap H_{1}\left([0, T]: L_{2}(\Omega)\right)$ and a $\gamma \in L_{1}([0, T] \times \Omega)$ such that

$$
\begin{gathered}
u_{t t}-\Delta u+\gamma=0 \text { in }[0, T] \times \Omega \\
\gamma \in \hat{g}(u) \quad \text { a.e. on }[0, T] \times \Omega . \\
\left.u\right|_{t=0}=\phi . \\
\partial u /\left.\partial t\right|_{t=0}=\psi
\end{gathered}
$$

This theorem provides a solution of the boundary value problem

$$
\begin{aligned}
& u_{t}-\Delta u+g(u)=0 \text { in }[0, T] \times \Omega, \\
& u=0 \text { on }[0, T] \times \partial \Omega
\end{aligned}
$$

with initial data given by (9), (10). The proof proceeds by standard techniques together with modification from $\$ 2$ needed to handle the fact that $g$ is discontinuous. One could also use the method mentioned before of choosing $u_{n}$ to be an exact solution of $u_{t t}-\Delta u+g_{n}(u)=0$ and then consider the limit $n \rightarrow \infty$ controlling the $g_{n}\left(u_{n}\right)$ term as in $\S 2$.

Similar results can be obtained for strongly nonlinear parabolic equations. The point is that with the right "sign condition" on $g$ one can obtain a weak solution provided one fills in the jumps of $g$ as in \$2. The emergence of multiple valued maps is an essential feature of discontinuous semilinear equations.

\section{REFERENCES}

1. H. Brézis, Opérateurs maximaux monotones et semi-groupes de contractions dans les espaces de Hilbert, North-Holland, Amsterdam, 1973. MR 50 \# 1060. 
2. P. McKenna and J. Rauch, Strongly nonlinear perturbations of nonnezatioe boundary value problems with kernel (to appear).

3. J. L. Lions, Quelque méthodes de résolutions des problèmes aux limites now linéaires, Dunod; Gauthier-Villars, Paris, 1969. MR 41 \#4326.

4. W. Strauss, On weak solutions of semilinear hyperbolic equations, An. Acad. Brasil. Ci. 42 (1970), 645-651.

Departugnt of Mathematics, University of Michioan, ANN Arbor, Michigan 48104

Current address: School of Mathematics, Institute for Advanced Study, Princeton, New Jersey 08540 